\title{
Procesos de formación inicial docente en contextos multiculturales: inclusión y exclusión*
}

\author{
Initial teacher training processes in multicultural context: inclusion and exclusion
}

Processos de formação docente inicial em contextos multiculturais: inclusão e exclusão

\author{
Orietta Fabiola Geeregat Vera1, Olga Angélica Vásquez Palma², Juan Manuel Fierro Bustos ${ }^{3}$ \\ ${ }^{1}$ Universidad de La Frontera, Facultad de Educación y Humanidades, Temuco, Chile, ogeerega@ufro.cl, \\ 56-045- 325388 \\ ${ }^{2}$ Universidad Católica de Temuco, Escuela Ciencias de la Salud, ovasquez@uct.cl, \\ 56-045- 553881 \\ ${ }^{3}$ Universidad de La Frontera, Facultad de Educación y Humanidades, Temuco, Chile, jmfierro@ufro.cl, \\ 56- 045- 32506
}

\begin{abstract}
RESUMEN
El artículo presenta una visión crítica de la formación inicial docente en contextos multiculturales; discute respecto a la posibilidad de existencia de una relación intercultural entre dos sociedades en que se manifiesta una abierta jerarquía entre ambas. Se sostiene que los resultados de este tipo de interrelación no sólo son incomunicantes, sino excluyentes desde su origen; este fenómeno es particularmente importante de ser considerado en la formación de profesores, situación en que se incuba la posibilidad de un cambio social. Se trata de generar una discusión epistemológica profunda que sitúe al paradigma de la ciencia occidental y su principal agente reproductor -el sistema educativo- en similar nivel de prestigio que los paradigmas de conocimiento indígena y sus formas de enseñar. Al observar este fenómeno en su origen: la formación inicial de profesores en un contexto territorial de relación permanente entre culturas diversas, se hace viable intentar resolver la pregunta si efectivamente es posible formar profesores(as) que impartan una educación intercultural, o con menor ambición, que posean las competencias para implementar una educación contextualizada al origen de sus estudiantes, o que, al menos, puedan conocer respecto al modo en que conocen los portadores un saber tradicional cuyos orígenes paradigmáticos se asientan en un mundo precolombino absolutamente ajeno a los sustentos de la ciencia proveniente de occidente.
\end{abstract}

Palabras clave: formación docente, interculturalidad, educación inclusiva.

\begin{abstract}
This paper presents a critical vision of the in multicultural contexts discussing the possibility of an open hierarchy related to the intercultural relationship between the major society and the subordinated one. The results of such relation are excluding and non-communicative from its origin; this situation becomes important in initial teaching training because that is the moment to incubate a possible social change. The idea is to promote a deep epistemological discussion which locates western paradigm and its main reproductive agent - the educational system - in an equivalent prestige to indigenous knowledge and its ways of teaching. When looking at this phenomenon from its origin, initial teaching training in a permanent territorial relationship, it is fair to ask if it is possible to form teachers to teach intercultural education, or less ambitious, teachers with tools to implement an education focused on the origin of their students, or who just show how indigenous students are inheritors of a pre-discovery culture far away from western foundation science.
\end{abstract}

Key words: action, motor functions, didactics, relationality.

\footnotetext{
* $\quad$ Productividad del Proyecto DI07-0036 (2007-2009)
} 


\section{RESUMO}

Apresenta-se visão crítica da formação inicial docente em contextos multiculturais; discute-se a respeito da possibilidade da existência de uma relação intercultural entre duas sociedades em que se manifesta uma aberta hierarquia entre ambas. Sustenta-se que os resultados deste tipo de inter-relação se comunicam, senão excludentes em suas origens; é importante que tal fenômeno seja considerado na formação de professores, onde se incuba a possibilidade de transformação social. Trata-se de gerar profunda discussão epistemológica que situe o paradigma da ciência ocidental e seu principal agente reprodutor - o sistema educativo - em similar nível de prestígio que os paradigmas de conhecimento indígena e suas formas de ensiná-lo. Ao observar este fenômeno em sua origem: a formação inicial de professores em um contexto territorial de permanente relação entre culturas diversas, viabilizando esclarecer sobre a possibilidade de, efetivamente, formar professores(as) para uma educação ou, em menores proporções, que possuam as competências para implementar uma educação contextualizada em que a origem dos estudantes sejam consideradas, ou que, no mínimo, possam conhecer como se conhecem os portadores de saberes tradicionais cujas origens paradigmáticas se assentam em um mundo pré-colombiano totalmente alheio aos sustentos da ciência ocidental.

Palavras chave: formação docente, interculturalidade, educação inclusiva.

\section{FORMACIÓN DOCENTE: INCLUSIÓN-EXCLUSIÓN}

Los procesos de formación docente se caracterizan por atender y asumir en los trayectos de formación diversas demandas sociales; una de ellas es responder a los desafíos de integración. En este ámbito, el currículum permanentemente se condiciona para contener a lo menos una visión general de los sujetos a quienes está dedicado. Este principio se tensiona cuando se asumen procesos de formación de actores educativos relevantes en contextos inter y multiculturales.

En los currículum nacionales y particularmente en los de la Novena Región, determinada por las condiciones de territorio de "frontera" y de componente humano multiétnico, es cada vez más explícito en los perfiles, programas y asignaturas pedagógicas la "atención a la diversidad". Lo anterior se expresa recurriendo a conceptos como: favorecer relaciones interétnicas e interculturales, atender a la diversidad, a la vulnerabilidad y a las necesidades especiales, además de considerar los contextos multiculturales, incorporar cosmovisión y lengua mapuche, describir situaciones de lenguas en contacto, revitalizar las culturas indígenas, entre otras.

Frente a las decisiones políticas, nacionales e institucionales locales, cabe preguntarse si los curriculum de formación de profesores, cuando se declaran inclusivos, están efectivamente realizando un proceso natural o impostado que surge a partir de relaciones asimétricas que se revelan en el lenguaje. Afirmaciones tales como: tolerar la diversidad, resistencia cultural, asimilar otras culturas, aceptar al otro como legítimo otro, son expresiones que en diversas esferas de discusión académica, dan cuenta de la existencia de una problemática social que niega la declaración de pluralidad que se erige como principio de diversos proyectos educativos universitarios.

De este modo, el fenómeno educativo y su presencia en contextos interculturales resulta abordable desde el punto de vista relacional, sistémico y complejo, en donde todos sus componentes se impactan e impactan al otro al interrelacionarse; desde este punto de vista, Maturana plantea que "todo ser vivo existe en interacciones en un medio. Lo que le pasa a este ser vivo en sus interacciones es que cada encuentro con el medio gatilla en él un cambio estructural particular, determinando en él en su estructura en el momento del encuentro. Cada encuentro del medio con el ser vivo gatilla en el medio un cambio estructural determinado en el medio. La consecuencia inevitable y espontánea 
de esto es que en una historia de interacciones recurrentes, ser vivo y medio cambian de una manera congruente" (1992: 229); al asumir la educación en contextos interculturales, como un proceso en que los aspectos más productivos son los fenómenos interrelacionales, de seres vivos que conviven en un ambiente con el fin último de conocer, resulta fundamental realizar la discusión respecto al producto cognitivo-cultural de este proceso, considerando, además, que existen patrones de dominio social que se expresan en la educación, como plantea Bourdieux (2003), en que ese nicho ecológico reproduce diferencias de clase y de poder y que dichas relaciones no representan un dominio de lo natural sino de lo creado por el ser humano de manera artificiosa; también sucede lo mismo con la educación como estructura y modelo la cual puede ser alterada por la intención manifiesta de la ideología que la sostiene.

\section{EL CONOCER EN LA EDUCACIÓN: UN PRODUCTO COGNITIVO-CULTURAL}

Considerando la importancia del fenómeno interrelacional en la educación, contextualizado en patrones de dominio cultural, y definiendo el resultado educativo como un producto cognitivo-cultural, resulta esencial asumir que los profesores, tanto en su formación inicial universitaria como en el ejercicio de su profesión, necesitan redireccionar el fenómeno del conocer, posicionando el objeto en sí mismo en primera instancia, y luego plantearse si es posible entablar diálogos fluidos con otro al cual se le pretende educar con un paradigma y contenidos epistémicos que no son los suyos.

La discusión se asienta entonces en develar el concepto de inclusión y relevar la urgente necesidad de enfrentar nuestra condición diversa desde su condición natural, además de propiciar la discusión analítica de la apuesta paradigmática de la educación chilena, partiendo del supuesto que todo saber es excluyente, en tanto que es un producto cultural en el cual se implican reglas y afirmaciones compartidas por una determinada comunidad y manifestadas por medio de una lengua común, la cual no incluye elementos simbólicos que no están presente en la construcción endémica de realidad. Lo anterior es una reflexión de primer orden para abordar las relaciones interculturales y, más aún, para abordar procesos de formación universitaria que declaren asumir la multiculturalidad como principio.

Ulin, comentando a Winch, plantea que "comprender una cultura indígena, o más bien cualquier cultura, implica necesariamente comprender las reglas intersubjetivas o los juegos de lenguaje de los cuales derivan su sentido tanto sus acciones como sus productos culturales" (1997: 16). En este sentido, el proceso de enseñanza de y a una cultura diversa implica tener la pretensión de conocer profundamente las reglas y significados que subyacen al fenómeno de construcción e interpretación de un mundo particular y sus formas de construir realidades intersubjetivas; de este modo se manifiesta lo que Ricoeur (1974) ha llamado conflicto de las interpretaciones.

La educación como disciplina está inserta en un modelo institucional y bajo ese marco responde a exigencias que emanan de un modelo de sociedad dominante, con un paradigma que impone su interpretación de realidad por sobre las otras; difícilmente esta propuesta educativa puede lograr comprender las reglas intersubjetivas o los juegos de lenguaje de las culturas diversas, más aún, resulta complejo que los miembros de una cultura diversa logren internalizar los contenidos propuestos a través de un proceso 
educativo auto-reflexivo y retroalimentador, por cuanto, se quiera reconocer o no la imposición de nuevos modos de construir realidad, produce en quien lo recibe fragmentación de contenidos y una ilusión de "saber del otro"; así se construye un ser fraccionado que superpone nuevas imágenes en su construcción identitaria, incorporando nuevos significados de una realidad ajena y, por lo mismo, su producto constituye a lo más una reinterpretación que satisface la necesidad de vencer la incertidumbre.

Lo anterior no niega, sino que afirma la realidad cambiante e inacabada del ser humano, quien incorpora de manera constante elementos de un contexto dinámico; por ello se pretende hacer un llamado de atención respecto de un fenómeno que se considera parte de un orden natural de las cosas, como lo es que miembros de una cultura diversa aprehendan significados, símbolos y una racionalidad en que subyace un paradigma de ciencia que no es el propio, y que no se reconozca que este paradigma de ciencia no necesariamente es el único y el verdadero, sino que es sólo una de las tantas formas de acceder al conocimiento de la realidad social y natural.

Esta reflexión invita a observar lo dominante que resulta nuestra forma de abordar la educación en un contexto cultural diverso y en lo arrogante que resulta pensar que podemos comprender al otro, conocerlo y, no conformes con ello, "educarlo"; decirle cuáles son las formas y modos correctos de pensar, sentir y actuar frente a determinados fenómenos. Se plantea que resulta muy complejo y curioso, desde esta perspectiva, abordar la interculturalidad o siquiera pensar que es posible, mas aún plantear que puede, en el contexto antes mencionado, promover principios justos y respetuosos de implementar una formación inicial docente que sea inclusiva con la diversidad.

Es necesario, como plantea Skliar (2002:15), "volver a mirar bien, es decir, volver la mirada más hacia la literatura que hacia los diccionarios, más hacia los rostros que hacia las pronunciaciones, más hacia lo innombrable que hacia lo nominado. Y seguir desalineados, desencajados, sorprendidos, para no seguir creyendo que "nuestro tiempo", "nuestro espacio", "nuestra cultura", "nuestra lengua", "nuestra mismidad" quiere decir "todo el tiempo", "todo el espacio", "toda la cultura", "toda la lengua", "toda la humanidad".

De este modo, resulta fundamental considerar al momento de diseñar un currículum educativo que contemple la formación de la pluralidad en sus principios, lo que ya en 1974 planteaba Winch en su teoría de juegos del lenguaje “... la necesaria relación entre reglas y significado revela que no hay una sola racionalidad sino, potencialmente, tantas racionalidades distintas como comunidades de hablantes (...) las racionalidad la constituye entonces la lógica informal de vida compartida por una comunidad de hablantes o una tradición cultural"; esta afirmación elemental, en la actualidad, no tiene los espacios y los tiempos para revelarse, ya que el sistema educativo actual, como plantea Bourdieu (1983), enseña y reproduce el paradigma que pertenece al grupo que ocupa una posición de poder en la estructura social; a través de este proceso, aquél que pertenece a las clases o grupos marginados debe conceder que son otros los que saben, aquéllos que detentan el dominio social, por lo mismo, considerando la visión de Foucault (2006), los marginados son obligados a negar su posibilidad de poder a través de la negación de su saber y en consecuencia negar la expresión de ser para, paradojalmente, acceder a un tipo de conocimiento que lo encarcela, lo excluye y lo margina en su verdadera identidad, y en su proyecto del ser, que niega y estigmatiza su origen familiar y contextual, otorgándole una posibilidad de posicionarse en el sistema sólo si admite su negación; ante ello cabe preguntarse, ¿es esto un sistema educativo inclusivo, pluralista y contextualizado? 
El sistema educativo enmascara la ideología que lo sustenta, esconde la falta de opciones a través de una falsa bondad que "incluye" en la negación del otro, como Bourdieu plantea (2003), el sistema educativo formal ("la escuela") impone un paradigma cultural de manera arbitraria reproduciendo la estructura social y las relaciones de clase, adjudicando posiciones de estatus a ciertos tipos de vestimentas, modos de hablar y de "consumir" educación, y por lo mismo, subordina a todo aquello que se manifiesta de modo diferente.

De acuerdo a lo anterior, el sistema escolar forma en las personas un proceso de adoctrinamiento que es la base de la reproducción cultural y social. Los que no adquieren esta formación son "excluidos", ya que el sistema les impone una cultura dominante, lo que implica renunciar a su propia cultura, en otras palabras, someterse a un conjunto de reglas, valores y creencias que muchas veces no son concordantes con su estilo de vida.

\section{CIENCIA HEGEMÓNICA: ¿EDUCACIÓN INCLUSIVA?}

La educación formal nos enseña a obedecer, establece la lealtad con el sistema como una condición de la "naturaleza humana"; desde la perspectiva de Bourdieu (1983), el sistema educativo imperante debe procurar la incorporación en el estudiante del capital cultural necesario, validado por la élite, asegurándose que el sujeto aprendiz internalice lo que es legitimado por el grupo que detenta el poder; si no lo hace, quedará excluido, en el margen.

Los contenidos reflejan una norma y esta norma no es sino una de las funciones del orden cultural establecido. Al ser parte del engranaje cultural, los contenidos organizados en un plan de estudios forman parte de un perfil profesional y obedecen a una práctica instrumental, sustentada en premisas que tienen la pretensión de ser verdaderas. En la actualidad, en el ámbito de la interrelación entre culturas, estas premisas otorgan mayor prestigio social y mejor estatus académico al conocimiento que proviene de Europa, en desmedro de lo que nace en nuestras tierras; pretendemos la generación de relaciones interculturales más horizontales, pero fundamos nuestra identidad en la autoexclusión y en la subordinación de un modo de conocer con respecto de otro; pese a ello en el discurso permanece el supuesto de estar incorporando en nuestro currículo educativo la educación inclusiva; decimos una cosa y hacemos otra y mantenemos la paradoja no sólo en las acciones sino también en el lenguaje; de este modo el contacto entre las estructuras del trabajo y las educativas aparece constantemente en oposición. Toda transmisión cultural se avizora perfectible, para que la acción humana pueda ensanchar y profundizar los niveles de conciencia, pero al mismo tiempo debe saber transformar esta acción y mejorar la experiencia que se transmite.

Asumir los párrafos precedentes, tal vez no es novedoso, ni alentador, pero el sentido profundo de hacerlos explícitos es encontrar en la naturaleza social y cultural de las cosas su carácter mítico e iniciar su reconstrucción. En el proceso desmitificador, el mito se reactualiza a través de los ritos y el ritual académico permite resignificar los contenidos, saber, conocer y hacer público los manifiestos, permite modificar, equilibrar y renovar las prácticas educativas.

La discusión presentada surge de la preocupación respecto del proceso educativo en nuestra sociedad y su impacto en la formación de las futuras generaciones y el vínculo con la sociedad que anhelamos, o aquélla que el sistema educativo está proyectando 
reproducir a través de la estimulación y desarrollo de ciertas competencias y saberes en el ser humano en desmedro de otras. Esta afirmación se sustenta en los resultados empíricos del Proyecto "Utopía y realidad: revelaciones de la formación inicial de profesores en la Universidad de La Frontera" (DI07- 0036), en el cual se concluye que el currículo escrito en relación con su puesta en práctica manifiesta distancias a considerar, principalmente en lo que se refiere a la articulación e integración de conocimiento entre las distintas disciplinas; ello coincide con la percepción que manifiestan tanto académicos como estudiantes entrevistados. Dicha desarticulación se manifiesta en una disputa disciplinaria continua donde se pretende establecer el dominio de un ámbito del conocimiento por sobre otros desperdiciando la oportunidad de apreciar en toda su complejidad los distintos saberes.

El fenómeno descrito se manifiesta en la formación inicial docente de manera aún más evidente en la expresión de dominio entre paradigmas culturales, abordándose la interculturalidad desde el sesgo del conocimiento científico occidental.

Las actuales soluciones o respuestas correctas frente a este fenómeno social son parte de un proceso enmascarado que mantiene las convenciones, los decretos y los objetivos altruistas de una igualdad mal entendida y menos discutida, subrayando, una vez más, la dominancia de quien la impone (todos iguales a mí).

Por lo mismo, como primer ejercicio, actualizamos las palabras de Skliar: "Que sigo pensando que para hablar de cambios en la educación es necesario, primero, un profundo silencio, una larga espera, una estética no tan pulcra, una ética más desalineada, dejarse vibrar por el otro más que pretender multiculturalizarlo, abandonar la homo-didáctica para hetero-relacionarse" (2002: 15).

Desde este punto de vista se puede señalar como alternativa pedagógica lo que autores como Walsh (2008) llaman la de-colonialidad, que se sustenta en una praxis que construya una conciencia de subjetividad reflexiva, incluyendo a educandos y educadores, la cual se constituye en un medio para visibilizar y desestructurar un modelo educativo en el que subyace un orden y lógica todavía sustentado en estructuras sociales, políticas y epistémicas coloniales: "estructura hasta ahora permanentes que mantienen patrones de poder enraizados en la racialización, en el conocimiento eurocéntrico y en la interiorización de algunos seres como menos humanos" (56). Dicha estructura responde a un lineamiento central de cómo se construye válidamente el conocimiento, situando a la ciencia como única fuente de acceso al conocimiento, siendo ésta "quien ha contribuido en forma vital al establecimiento y mantenimiento del histórico y actual orden jerárquico racial, en el cual los blancos, y especialmente los hombres blancos europeos, permanecen en la cima" (56). El desafío es construir nuevos marcos epistemológicos que efectivamente permitan la visualización de una realidad diversa, incluyente y sorprendente que nos interrogue permanentemente respecto a los marcos establecidos.

De este modo, para la proyección e implementación de una propuesta de saber inclusivo e incluyente, es necesario el cambio paradigmático que se traduzca y exprese procedimental y actitudinalmente, para permitir el abordaje y la constitución de un sujeto integral, multidimensional, validado en toda su naturaleza, con una visión de humildad que reconozca la diversidad cultural inherente a ser humano y sus productos intelectuales, espirituales y materiales. 


\section{REFERENCIAS BIBLIOGRÁFICAS}

Bourdieu y Passeron. (1972). La reproducción. Elementos para una teoría del sistema de enseñanza. Barcelona: Ed. Laia.

Bourdieu, P. (1983). Campo de poder y campo intelectual. Buenos Aires: Folios Ediciones.

Bourdieu, P. (2003). Capital cultural, escuela y espacio social. México: Siglo Veintiuno Editores.

Durkheim E. (1994). Las reglas del método sociológico. Madrid: Alianza.

Foucault, M. (2006). Vigilar y castigar. Argentina: Siglo XXI.

Maturana, H. (1992). El sentido de lo humano. Santiago: Ediciones Pedagógicas Chilenas.

Ricoeur, Paul. (1974). The conflict of interpretations: essays in hermeneutics. Evanston: Northwestern University Press.

Ulin, Robert (1997). Antropología y Teoría Social. México: Siglo XXI Editores.

Walsh, Catherine (2008). "Interculturalidad crítica. Pedagogía de-colonial" en Diversidad, interculturalidad y construcción de ciudad (pp. 44-63). Colombia: Universidad Pedagógica Nacional. 
